\title{
Modulation of angiogenesis by tumor associated macrophages in the tumor microenvironment
}

\begin{abstract}
Macrophages are important cells in wound healing, providing aids for tissue cell growth, tissue matrix remodeling and angiogenesis. Solid tumor comprises not only tumor cells, but also tissue matrix and many stromal cells such as fibroblasts and macrophages. Tumor associated macrophages (TAMs) promote cancer metastasis through several mechanisms including tumor angiogenesis, tumor growth and cell migration and invasion. Complex paracrine signaling networks exist between TAMs and cancer cells to activate each other. The colony stimulating factor 1/epidermal growth factor paracrine loop is well known of breast cancer cell invasion. TAMs derived protease, such as matrix metalloproteinases, urokinase-type plasminogen activator, and cathespin B can promote cancer cell metastasis. By interacting with cancer cells, TAMs can be induced to express more cytokines and tissue matrix-degrading enzymes, such as matrix metalloproteinases, plasminogen activators and cathepsin $\mathrm{B}$, that are either direct angiogenic factors or tissue matrix modulators responsible for promoting tumor angiogenesis. With evidence indicating TAMs tumor-promoting roles, many investigations have been undertaken to study the potential of using TAMs as the therapeutic target while some conflicting investigations have demonstrated their role in anti-tumor activity. More detailed mechanisms regarding the interaction between TAMs and tumor still need to be explored, and a thorough understanding of this interaction will provide some helpful conceptual suggestions for future cancer therapy. This mini review will summarize information about the role of TAMs on modulating angiogenesis in the inflammatory micro-environment of solid tumors and discuss the potential targets for future therapeutic approaches.
\end{abstract}

Volume I Issue $3-2014$

\author{
Joseph F Murphy \\ National University of Ireland, Ireland
}

Correspondence: Joseph F Murphy, National University of Ireland, Ireland,Tel 205-500-8990,Email drjfmurphy@yahoo.com

Received: July 01, 2014 | Published: July 24, 2014
Abbreviations: TAM, tumor associated macrophage; MCP, monocyte chemotactic protein; GMCSF, granulocyte macrophage colony stimulating factor; HIF, hypoxia-inducible factor; PyMT, polyoma virus middle $t$ oncoprotein; VEGF, vascular endothelial growth factor; bFGF, basic fibroblast growth factor; EGF, epidermal growth factor; TGF- $\alpha$, transforming growth factor- $\alpha$

\section{Introduction}

The inflammatory infiltrate in most cancers contains several cell types, including lymphoid cells, granulocytes, mast cells, dendritic cells, natural killer cells and macrophages, which are the most highly represented cell type. Macrophages are associated with the development and progression of a variety of tumors, including breast, prostate, glioma, lymphoma, bladder, lung, cervical and melanoma. In breast cancer, macrophages have been reported to comprise up to $50 \%$ of the tumor cell mass. ${ }^{1}$ An important inflammatory cell type commonly found in the stroma of tumor is the tumor- associated macrophage (TAM2). ${ }^{2}$ In normal immune system, macrophages play an important role in wound healing. They provide aids for tissue matrix remodeling, cell growth and angiogenesis. ${ }^{3,4}$ Similar to macrophages in wound healing, TAMs play an important role in tumor progression by exerting their multiple functions in tumor cell proliferation, tumor cell invasion, and tumor angiogenesis. ${ }^{5}$ When a solid tumor grows beyond $2 \mathrm{~mm}$ in diameter, simple diffusion of oxygen and nutrients to metabolizing tissues becomes insufficient.

One of the common features of solid tumors is hypoxia is a common feature of solid tumors. Some of these factors, such as monocyte chemotactic protein-1 (MCP-1) and granulocyte-macrophage colonystimulating factor (GMCSF), are potent chemokines chemotactic toward the monocytes in nearby blood vessels. ${ }^{6}$ Monocytes are thus continually recruited into tumors, differentiated into TAMs, and then accumulate in the hypoxic areas. These cells have been considered to have dual effects on the tumor, i.e. anti-tumor and pro-tumor effects. They exhibit tumoricidal activity toward tumor cells at initial contact, but when they reside in the areas of hypoxia, macrophages respond to hypoxia by up-regulating transcriptional factors like hypoxia-inducible factors 1 and 2 (HIFs 1 and 2), which in turn activate a broad array of genes for promoting tumor cell invasion and tumor angiogenesis. ${ }^{7}$ Eventually, TAMs function as the provider of tumorigenic factors as well as regulators for malignant development. Accumulating evidence has suggested that high TAM infiltration is advantageous to tumor spreading in certain cancers, most notably via enhancement of tumor angiogenesis and tumor cell migration and invasion, ${ }^{8}$ which could explain why high levels of TAMs correlate with poor prognosis in these cancers. ${ }^{9}$

\section{Clinical association of TAM with tumor} angiogenesis in human cancers

It is well established that the majority of malignant tumors contain numerous macrophages as a major component of the host leukocytic infiltrate. ${ }^{10}$ These cells were originally thought to be part of the host immune system used to defend the tumor cells; however, many clinical investigations have indicated the correlation between high TAM level and worse prognosis in human cancers. Several studies have elucidated studies of macrophage infiltration in various human cancers and its relationships with tumor angiogenesis. Several human cancers, such as breast, ${ }^{11}$ prostate, ${ }^{12}$ uterine cervical, ${ }^{13}$ uterine endometrial, ${ }^{14} \operatorname{liver}^{15}$ have been reported to display significant correlation of macrophage infiltrating level with tumor angiogenesis. Macrophage infiltration is also well associated with poor prognoses in these cancers. ${ }^{9}$ In addition to the clinical correlation between increased TAM level and higher vascular density, the pro-angiogenic function 
of TAMs has been demonstrated by experimental mouse models. ${ }^{5}$ In polyoma virus middle $\mathrm{T}$ oncoprotein (PyMT)-induced mouse mammary tumors, macrophages were recruited to the lesions where angiogenesis subsequently occurred before transition into malignant sites. Depletion of macrophages in PyMT-induced tumors resulted in a $\sim 50 \%$ decrease of vascular density and thus the delay of malignant transition. In contrast, enhanced recruitment of macrophages by over expression of colony-stimulating factor- 1 (CSF-1) resulted in accelerated vascularization and progression to malignancy. ${ }^{16}$

Bingle et al. ${ }^{17}$ studied the effect of macrophages on tumor angiogenesis by using a mouse dorsal skin fold chamber model, which allowed direct in situ visualization and monitoring of implanted tumor spheroids and the surrounding blood vessels in vivo. Their results indicated that the presence of macrophages in tumor spheroids resulted in at least a 3-fold increase of VEGF release and significantly greater angiogenesis.

\section{Mechanistic studies for macrophages- associated angiogenesis}

The increasing body of evidence has indicated that tumor angiogenesis was induced not only by the tumor cells but also the TAMs. ${ }^{18-21}$ In vitro studies have also shown that TAMs are capable of producing a broad spectrum of angiogenesis-modulating factors including growth factors, cytokines and tissue matrix-degrading enzymes. ${ }^{22-24}$ Growth factors: A number of growth factors, including vascular endothelial growth factor (VEGF), basic fibroblast growth factor (bFGF), epidermal growth factor (EGF), and transforming growth factor- $\alpha$ (TGF- $\alpha$ ), are known to be produced by TAMs. They are not only growth factors for tumor cells, but also potent mitogens to promote endothelial cell proliferation. ${ }^{25}$ Among them, VEGF is well-documented angiogenic factor that can promote endothelial cell growth, maturation and survival in a range of experimental systems. ${ }^{26}$ VEGF is strongly expressed in glioblastoma, the most frequent brain malignancy, which is characteristically highly vascularised. ${ }^{27}$ Up-regulation of VEGF has also been observed in human breast malignancy, and correlated with microvessel density in invasive breast carcinoma. ${ }^{28}$ VEGF also functions as a monocyte chemotactic factor to attract TAMs to the hypoxic areas of tumor. In addition, VEGF can cause persistent extravasation of fibrin and fibronectin and continuous generation of tissue matrix. ${ }^{1}$

\section{Cytokines}

Several inflammatory cytokines have been known to participate in macrophages associated angiogenesis during the wound healing process. ${ }^{22,23}$ Interleukin-8 (IL-8) is mitogenic to endothelial cells and stimulates angiogenesis in animals..$^{29}$ The conditioned media of macrophages have been observed to achieve the same effects, and the effects can be markedly abolished by anti-IL- 8 antibody, suggesting that IL- 8 produced by macrophages plays an important role in macrophage-associated angiogenesis. ${ }^{30}$

Tumor necrosis factor- $\alpha$ (TNF- $\alpha)$ is also one of the major cytokines involved in macrophage-associated angiogenesis. TNF- $\alpha$ was postulated to be used by macrophages to kill cancer cells and cause tumor necrosis. When over expressed in endothelial cells, TNF- $\alpha$ was growth-inhibitory and even cytotoxic to endothelial cells. ${ }^{31,32}$ In contrast, exogenous application of TNF- $\alpha$ promoted formation of new blood vessels in several in vivo models. ${ }^{33,34}$ Leibovich et al. ${ }^{35}$ further showed that anti-TNF- $\alpha$ antibodies completely neutralized the angiogenic activity in the conditioned media of activated macrophages, supporting the role of TNF- $\alpha$ as an angiogenic molecule from macrophages. Moreover, macrophages are an important source of interleukin-6 (IL-6). IL-6 is involved in macrophage-mediated wound healing and, although evidence revealing its angiogenic activity is still incomplete, a recent study has demonstrated its role in tumor angiogenesis for colon cancer cells. ${ }^{36}$

\section{Multiple factors involved in tams-associated tumor angiogenesis}

For solid tumors, tumor angiogenesis is an essential process for continuous tumor growth and spreading. Endothelial cell's biomechanical properties are regulated by invasive cancer cells. ${ }^{37}$ Tumor angiogenesis can be regulated by a complex interaction between cancer cells and TAMs that produces angiogenic factors and tissue matrix modulating factors. Angiogenic factors exert direct effects on endothelial cells and tissue matrix is also involved in regulating angiogenesis. ${ }^{38}$ It is a supporting matrix for vessels but a barrier for protruding or migrating endothelial cells Tissue matrix is also a reservoir of angiogenic factors. Many angiogenic factors, such as VEGF, bFGF and TGF- $\beta$, have an affinity for heparin and thus are sequestered by tissue matrix by binding to heparin like glycosaminoglycans. Tissue matrix modulating factors are not only responsible for tissue matrix remodeling; they are also capable of regulating the release of matrix-binding angiogenic factors following the degradation of tissue matrix. It has long been established that endothelial cells can be induced to display the morphology of capillary tubes and form a network structure on Matrigel, which can be an in vitro marker of angiogenesis. ${ }^{39} \mathrm{VEGF}$ is a typical potent inducer in this assay system. Moreover, it has been reported that interaction between cancer cells and macrophages can produce sufficient factors for promoting endothelial cell network formation. Furthermore, this effect of cancer cells/macrophages interaction on endothelial cells can be inhibited by incubation with either anti-IL-8 receptor antagonizing antibody or IL-6 or TNF- $\alpha$ soluble receptor, suggesting that IL-8, TNF- $\alpha$ and IL- 6 indeed participate in TAMs related angiogenesis. ${ }^{40}$

\section{Tissue matrix-degrading proteinases}

Macrophages are a rich source of tissue matrix-degrading enzymes and inhibitors. They are capable of producing MMPs (e.g. MMP-2, MMP-7, MMP-9, and MMP-12), serine proteinases (such as plasminogen activators uPA and tPA), cysteine proteinase (e.g. cathepsin $\mathrm{B}$ ), and plasminogen activator inhibitors to modulate the destruction and re-construction of tissue matrix]. Once the tissue matrix is proteolytically degraded, these factors are released and become angiogenic. Furthermore, some proteolytic products of tissue matrix, e.g. fibrin of plasmin and fragments of hyaluronic acid, can also facilitate angiogenesis. ${ }^{41,42}$

\section{TAMs as the target for anti-cancer studies}

Tumor angiogenesis is one of the major mechanisms by which TAMs exert their tumor-promoting activity, and therefore agents that can antagonize the angiogenic factors secreted by TAMs would be obviously effective in the inhibition of TAMs-induced angiogenesis. ${ }^{43}$ Furthermore, several studies have been undertaken to investigate the possibilities of suppressing secretion of angiogenic factors by TAMs and blocking macrophage infiltration into the tumor mass as anticancer strategies. For example, a low dose of interferon- $\gamma$ has been shown to inhibit secretion of TNF- $\alpha$ by macrophages. ${ }^{44}$ The compound chloroquine inhibits TNF- $\alpha$ gene transcription in macrophages. ${ }^{45}$ Chemokine inhibitors affect tumor growth by decreasing TAM infiltration. ${ }^{46}$ Moreover, transplanted tumors transfected with 
Interleukin- 10 show markedly less macrophage infiltration than their non-transfected counterparts. ${ }^{47}$ The anti-angiogenic agent linomide, by suppressing functions of macrophages, exhibits its efficacy to significantly reduce tumor volume in a murine prostate cancer model. ${ }^{48}$ Legumain, a member of the asparaginyl endopeptidase family, is over expressed by TAMs and thus serves as a target molecule. A legumain-based DNA vaccine was shown to drastically reduce TAM density in tumor and resulted in a marked decrease in angiogenic factors produced by TAMs. ${ }^{49}$ With the seemingly important role of TAMs in not only tumor angiogenesis but also tumor cell migration/ invasion, the TAMs themselves become an appealing target for cancer therapeutic approaches. One such approach is photodynamic therapy, which combines a non-toxic photosensitizer with harmless visible light of the specific wavelength to excite photosensitizer to a highenergy triplet state that will subsequently generate reactive oxygen species to kill target cells. Studies have reported specific targeting of photosensitizer to macrophages via conjugation of a photosensitizer to a ligand of the scavenger receptor class $\mathrm{A}$, and the illumination was confined to the tumor. It was assumed that only TAMs would be killed, and circulating monocytes and macrophages in other tissues might still have beneficial antitumor effects such as extinguishing micro metastasis. ${ }^{50,51}$ Because TAMs migrate and accumulate in hypoxic areas of tumor, they may serve as vectors to carry genes or drugs into these sites to be activated by hypoxia. ${ }^{52}$ The potential of developing such a delivery strategy is to overcome the problem that most drugs or gene therapy vectors are difficult to reach the tumor sites with low or even no vascular density. Antibody-dependent cell phagocytosis (ADCP) represents another promising approach and methods to enhance efficacy of this therapeutic modality have been described..$^{53}$ Another approach described by Carta et al. ${ }^{54}$ where they transduced macrophages with a viral vector containing IFN- $\gamma$ gene that was activated only in low oxygen conditions. Finally, advances in antibody engineering may represent another avenue. Recent results results highlighting the plasticity of TAMs, which are capable of promoting tumor progression and invasion while still retaining tumoricidal function in the presence of tumor-targeting mAbs, represents another promising approach to TAM mediated activity within the tumor microenvironment. ${ }^{55,56}$

\section{Summary}

TAMs are derived from peripheral blood monocytes recruited into the tumor and have multiple effects on tumor progression. They exhibit tumoricidal activity toward one fraction of cancer cells. Upon affected by both tumor cells and microenvironment, the TAMs can release a vast diversity of growth factors, cytokines, proteolytic enzymes, and other inflammatory modulators. With these factors, TAMs can induce tissue matrix remodeling and angiogenesis, and simultaneously enhance tumor cell migration and invasion, ultimately resulting in tumor metastasis. Clinically, the TAM level is significantly correlated with poor prognosis in several human cancers. As accumulating evidence indicates TAM's to tumor promoting roles, many investigations have been undertaken to study the potential of using TAMs as the therapeutic target. Targeting of macrophages in tumors is a promising therapeutic strategy. The depletion of TAMs or their 're-education' as anti-tumor effectors is under clinical investigation and may ultimately contribute to the success of conventional anti-cancer treatments.

\section{Acknowledgements}

The author is grateful to Tara Finn for the careful reading of this manuscript.

\section{Conflicts of Interest}

There is no conflict of interest.

\section{Funding}

None.

\section{References}

1. Mukhtar RA, Nseyo O, Campbell MJ, et al. Tumor-associated macrophages in breast cancer as potential biomarkers for new treatments and diagnostics. Expert Rev Mol Diagn. 2011;11(1):91-100.

2. Balkwill F, Mantovani A. Inflammation and cancer: back to Virchow? Lancet. 2001;357(9255):539-545.

3. DiPietro LA, Burdick M, Low QE, et al. MIP-1 alpha as a critical macrophage chemoattractant in murine wound repair. $J$ Clin Invest. 1998;101(8):1693-1698.

4. Martin P, Leibovich SJ. Inflammatory cells during wound repair: the good, the bad and the ugly. Trends Cell Biol. 2005;15(11): 599-607.

5. Guo C, Buranych A, Sarkar D, et al. The Role of tumor associated macrophages in tumor vascularization. Vasc Cell. 2013;5(1):20.

6. Murdoch C, Giannoudis A, Lewis CE. Mechanisms regulating the recruitment of macrophages into hypoxic areas of tumors and other ischemic tissues. Blood. 2004;104(8):2224-2234.

7. Lewis $\mathrm{C}$, Murdoch $\mathrm{C}$. Macrophage responses to hypoxia. Implications for tumor progression and anti-cancer therapies. Am J Pathol. 2005; 167(3):627-635

8. Shih JY, Yuan A, Chen JJ-W, et al. Tumor-associated macrophage: its role in cancer invasion and metastasis. J Cancer Mol. 2006; 2(3):101106.

9. Biswas SK, Allavena P, Mantovani A. Tumor-associated macrophages: functional diversity, clinical significance, and open questions. Semin Immunopathol. 2013;35(5):585-600.

10. Eljaszewicz A, Wiese M, Helmin-Basa A, et al. Collaborating with the enemy: function of macrophages in the development of neoplastic disease. Mediators Inflamm. 2013:831387.

11. Leek RD, Lewis CE, Whitehouse R, et al. Association of macrophage infiltration with angiogenesis and prognosis in invasive breast carcinoma. Cancer Res. 1996;56(20):4625-4629.

12. Lissbrant IF, Stattin P, Wikstrom P, et al. Tumor associated macrophages in human prostate cancer: relation to clinicopathological variables and survival. Int J Oncol. 2000;17(3):445-451.

13. Fujimoto J, Sakaguchi H, Aoki I, et al. Clinical implications of expression of interleukin 8 related to angiogenesis in uterine cervical cancers. Cancer Res. 2000;60(10):2632-2635.

14. Hashimoto I, Kodama J, Seki N, et al. Macrophage infiltration and angiogenesis in endometrial cancer. Anticancer Res. 2000;20(6c):48534856.

15. Peng SH, Deng H, Yang JF, et al. Significance and relationship between infiltrating inflammatory cell and tumor angiogenesis in hepatocellular carcinoma tissues. World J Gastroenterol. 2005;11(41):6521-6524.

16. Aharinejad S, Paulus P, Sioud M, et al. Colony-stimulating factor-1 blockade by antisense oligonucleotides and small interfering RNAs suppresses growth of human mammary tumor xenografts in mice. Cancer Res. 2004;64(15):5378-5384.

17. Bingle L, Lewis CE, Corke KP, et al. Macrophages promote angiogenesis in human breast tumour spheroids in vivo. Br J Cancer. 2006; 94(1):101-107. 
18. Lewis CE, Leek R, Harris A, et al. Cytokine regulation of angiogenesis in breast cancer: the role of tumor-associated macrophages. J Leukoc Biol. 1995;57(5):747-751.

19. Lee AH, Happerfield LC, Bobrow LG, et al. Angiogenesis and inflammation in invasive carcinoma of the breast. $J$ Clin Pathol. 1997;50(8):669-673.

20. Pollard JW. Tumour-educated macrophages promote tumour progression and metastasis. Nat Rev Cancer. 2004;4(1):71-78.

21. Condeelis J, Pollard JW. Macrophages: obligate partners for tumor cell migration, invasion, and metastasis. Cell. 2006;124(2): 263-266.

22. Sunderkotter C, Goebeler M, Schulze-Osthoff K, et al. Macrophagederived angiogenesis factors. Pharmacol Ther. 1991;51(2): 195-216.

23. Leek RD, Lewis CE, Harris AL. The role of macrophages in tumour angiogenesis. In: Bicknell R, Lewis CE, Ferrara N (Eds.), Tumor angiogenesis. Oxford, New York, USA. 1997;p. 81-99.

24. Sica A, Schioppa T, Mantovani A, et al. Tumour-associated macrophages are a distinct M2 polarised population promoting tumour progression: potential targets of anti-cancer therapy. Eur J Cancer. 2006;42(6):717727.

25. Bicknell R, Harris AL. Novel growth regulatory factors and tumor angiogenesis. Eur J Cancer. 1991;27(6):781-785.

26. Ferrara N, Gerber HP, LeCouter J. et al. The biology of VEGF and its receptors. Nat Med. 2003;9(6):669-676.

27. Plate $\mathrm{KH}$, Breier G, Weich HA, et al. Vascular endothelial growth factor is a potential tumor angiogenesis factor in human gliomas in vivo. Nature. 1992;359(6398):845-848.

28. Vicioso L, Gonzalez FJ, Alvarez M, et al. Elevated serum levels of vas associated macrophages in primary breast cancer. Am J Clin Pathol. 2006;125(1):111-118

29. Hu DE, Hori Y, Fan TP. Interleukin-8 stimulates angiogenesis in rats. Inflammation. 1993;17(2):135-143.

30. Koch AE, Polverini PJ, Kunkel SL, et al. Interleukin-8 as a macrophage derived mediator of angiogenesis. Science. 1992;258(5089):1798-1801.

31. Sato N, Goto T, Haranaka K, et al. Actions of tumor necrosis factor on cultured vascular endothelial cell: morphologic modulation, growth inhibition, and cytotoxicity. J Natl Cancer Inst. 1986;76(6):1113-1121.

32. Robaye B, Mosselmans R, Fiers W, et al. Tumor necrosis factor induces apoptosis (programmed cell death) in normal endothelial cells in vitro. Am J Pathol. 1991;138(2):447-453.

33. Frater-Schroder M, Risau W, Hallmann R, et al. Tumor necrosis factor type $\alpha$, a potent inhibitor of endothelial cell growth in vitro, is angiogenic in vivo. Proc Natl Acad Sci USA. 1987;84(15):5277-5281.

34. Rosenbaun JT, Howes EL Jr, Rubin RM, et al. Ocular inflammatory effects of intravitreally-injected tumor necrosis factor. Am J Pathol. 1988;133(1):47-53

35. Leibovich SJ, Polverini PJ, Shepard HM, et al. Macrophage-induced angiogenesis is mediated by tumour necrosis factor- $\alpha$. Nature. 1987; 329(6140):630-632.

36. Nagasaki T, Hara M, Nakanishi H, et al. Interleukin-6 released by colon cancer-associated fibroblasts is critical for tumour angiogenesis: antiinterleukin-6 receptor antibody suppressed angiogenesis and inhibited tumour-stroma interaction. Br J Cancer. 2014;110(2):469-778.

37. Mierke CT. Endothelial cell's biomechanical properties are regulated by invasive cancer cells. Mol Biosyst. 2012;8(6):1639-1649.
38. Fang M, Yuan J, Peng C, Li Y. Collagen as a double-edged sword in tumor progression. Tumour Biol. 2014;35(4):2871-2882.

39. Folkman J, Haudenschild C. Angiogenesis by capillary endothelial cells in culture. Trans Ophthamol Soc U K. 1980;100(3):346-353.

40. Lee $\mathrm{C}-\mathrm{C}$, Liu $\mathrm{K}-\mathrm{J}$, Chen $\mathrm{L}-\mathrm{L}$, et al. Tumor necrosis factor- $\alpha$, interleukin-8 and interleukin-6 are involved in vascular endothelial cell capillary tube and network formation induced by tumor-associated macrophages. J Cancer Mol. 2006;2(4):155-160.

41. Thompson WD, Campbell R, Evans T. Fibrin degradation and angiogenesis: quantitative analysis of the angiogenic response in the chick chorioallantoic membrane. J Pathol. 1985;145(1):27-37.

42. West DC, Hampson IN, Arnold F, et al. Angiogenesis induced by degradation products of hyaluronic acid. Science. 1985;228(4705):1324 1326.

43. Wahl LM, Kleinman HK. Tumor-associated macrophages as targets for cancer therapy. J Natl Cancer Inst. 1998;90(21): 1583-1584.

44. Pighetti GM, Sordillo LM. Regulation of mammary gland macrophage tumor necrosis factor-alpha production with interferon gamma. Res Vet Sci. 1994;56(2):252-255.

45. Zhu X, Ertel W, Ayala A, et al. Chloroquine inhibits macrophage tumor necrosis factor-alpha mRNA transcription. Immunology. 1993;80(1):122-126.

46. Robinson SC, Scott KA, Wilson JL, et al. A chemokine receptor antagonist inhibits experimental breast tumor growth. Cancer Res. 2003;63(23):8360-8365

47. Richter G, Kruger-Krasagakes S, Hein G, et al. Interleukin-10 transfected into Chinese hamster ovary cells prevents tumor growth and macrophage infiltration. Cancer Res. 1993;53(18):4134-4137.

48. Joseph IB, Isaacs JT. Macrophage role in the anti-prostate cancer response to one class of antiangiogenic agents. $J$ Natl Cancer Inst. 1998;90(21):1648-1653.

49. Luo Y, Zhou H, Krueger J, Kaplan C, et al. Targeting tumor associated macrophages as a novel strategy against breast cancer. $J$ Clin Invest. 2006;116(8):2132-2141

50. Demidova TN, Hamblin MR. Macrophage-targeted photodynamic therapy. Int J Immunopathol Pharmacol. 2004;17(2): 117-126.

51. Hamblin MR, Miller JL, Ortel B. Scavenger-receptor targeted photodynamic therapy. Photochem Photobiol. 2000;72(4):533-540.

52. Griffiths L, Binley K, Iqball S, Kan O, et al. The macrophage - a novel system to deliver gene therapy to pathological hypoxia. Gene Ther. 2000;7(3):255-262.

53. Nagashima H, Ootsubo M, Fukazawa M, et al. Enhanced antibodydependent cellular phagocytosis by chimeric monoclonal antibodies with tandemly repeated Fc domains. J Biosci Bioeng. 2011;111(4):391396

54. Carta L, Pastorino S, Melillo G, et al. Engineering of macrophages to produce IFN- $\gamma$ in response to hypoxia. $J$ Immunol. 2001;166(9):5374 5380 .

55. Grugan KD, McCabe FL, Kinder M, et al. Tumor-associated macrophages promote invasion while retaining $\mathrm{Fc}$-dependent antitumor function. J Immunol. 2012;189(11):5457-5466.

56. Kinder M, Greenplate AR, Grugan KD, et al. Engineered proteaseresistant antibodies with selectable cell-killing functions. J Biol Chem. 2013;288(43):30843-30854. 